\title{
La carte d'état-major des inventions brevetées : un avantage concurrentiel majeur pour innover
}

\section{Ordnance maps for patented inventions: a major competitive advantage} for innovation

\author{
Frédéric Caillaud ${ }^{1}$, Clément Sternberger ${ }^{2}$ \\ ${ }^{1}$ Institut National de la Propriété Industrielle (INPI), France, ffcaillaud@gmail.com \\ ${ }^{2}$ Institut National de la Propriété Industrielle (INPI), Université Paris-Saclay, RITM, France, csternberger@inpi.fr
}

\begin{abstract}
RÉSUMÉ. Le brevet est un marqueur précoce de l'innovation mondiale. Les dépôts se sont accrus de manière parfois exponentielle depuis une décennie permettant de constituer des bases de données pouvant en contenir jusqu'à 130 millions aujourd'hui. Constituées d'un empilement de sources et de qualité très différentes, ces bases sont difficilement exploitables pour mesurer de manière fiable la distance sémantique entre les concepts de familles de brevets et les visualiser sous forme de cartes pertinentes. Nous avons sélectionné la base DWPI et les algorithmes de traitements qui y sont associés pour créer des cartes des innovations mondiales et montrer, à travers différents exemples, comment les experts des grandes entreprises les utilisent. Ces cartes topographiques permettent d'identifier l'émergence des nouvelles innovations et les tendances mondiales, de positionner les inventions des entreprises dans leur environnement concurrentiel ce qui est essentiel pour arbitrer les investissements, sélectionner les partenaires les plus adaptés et accroitre, in fine, la productivité de la R\&D. C'est un outil indispensable pour maitriser l'Intelligence Technologique et mieux comprendre pourquoi les grandes entreprises ne communiquent pas sur le sujet afin de conserver un avantage concurrentiel majeur.

ABSTRACT. The patent is an early marker of global innovation. Repositories have grown exponentially over the past decade, making it possible to build databases that can hold up to 130 million today. As they are made up of a stack of very different sources and quality, these databases are difficult to use to accurately measure the semantic distance between the concepts of patent families and visualize them in the form of relevant maps. We have selected the DWPI database and the associated processing algorithms to create maps of global innovations and show, through various examples, how experts from large companies use them. These maps make it possible to identify the emergence of innovations and global trends, in order to position the inventions of companies in their competitive environment, which is essential to arbitrate investments, select the most suitable partners and ultimately increase R\&D productivity. It is essential to master Technological Intelligence and better understand why companies do not communicate on the subject, in order to maintain a major competitive advantage.

MOTS-CLÉS. Brevet, technologie, cartographie sémantique, innovation.

KEYWORDS. patent analytics, technology, mapping, innovation.
\end{abstract}

\section{Introduction}

Les responsables $R \& D$ ont tous le même problème : ils reçoivent des propositions d'inventions que leurs chercheurs qualifient généralement d'innovantes et d'originales et investissent dans leur développement ... alors que des inventions similaires ont déjà été brevetées quelque part dans le monde et que ces brevets pourraient leur interdire toute liberté d'exploitation commerciale sauf acquisition d'onéreuses licences. A leur décharge, il faut savoir qu'à ce jour, cent trente millions de brevets ont été déposés, que la croissance mondiale des dépôts de brevets s'est énormément accélérée depuis trente-cinq ans (elle est récemment devenue exponentielle en Chine) et que plusieurs millions de nouvelles inventions sont brevetées chaque année, dans des langues très différentes ([VAN 08, WIP 18]).

Ceci explique pourquoi aucun chercheur public ou privé, même vigilant, ne peut disposer aujourd'hui d'une vision précise des inventions concurrentes sans une analyse approfondie de ces 130 millions de brevets. Cette analyse doit être conduite le plus tôt possible afin de sécuriser les 
investissements qui seront mobilisés pour le développement et la valorisation des produits ([CHO 18], [MCM 03]). Cette analyse conduira soit à un abandon du projet, soit à une modification de ses spécifications et fonctionnalités, soit à la mise en place d'une collaboration avec le partenaire disposant des brevets nécessaires. Cette analyse permettra également de visualiser les orientations stratégiques de la R\&D des compétiteurs (entreprises, organismes publics de recherche - OPR - ou inventeurs indépendants) et de les contrecarrer en déposant de nouveaux brevets, comme on le ferait dans un jeu de Go.

Certains produits, notamment dans les technologies de l'information et les télécommunications, peuvent nécessiter des dizaines, voire des milliers de brevets pour être commercialisés sans risque de litige. Créer de tels pools de brevets nécessite une vision aussi précise que possible du positionnement des brevets afin d'identifier ceux qui sont nécessaires et ceux qui ne seront pas utiles. Les enjeux commerciaux sont si importants que de telles analyses conduisent à de nombreux litiges généralement très médiatisés. Il faut imaginer le monde des brevets comme un vaste jeu de Go mondial où le brevet est un pion, mais où les joueurs sont quelques dizaines de milliers dont certains, avec des forces de frappe colossales, peuvent créer de vastes champs de mines de brevets pour tenir à distance toute la concurrence. A titre d'exemple, L'Oréal dépose plus de brevets que tous ses concurrents majeurs réunis. Les entités qui ne disposent pas de tels moyens (notamment la recherche publique) doivent être particulièrement prudentes. La connaissance approfondie de son environnement en brevets est aussi importante que la créativité, pour survivre et se développer sereinement. La seule façon de réussir aujourd'hui est de réduire l'asymétrie d'information en analysant finement le contenu des bases de brevets [CAI 14]. Malheureusement, très rares sont les entités publiques ou privées capables de conduire des études suffisamment approfondies pour décider en connaissance de cause. On n'imagine pas un général d'armée partir en guerre sans avoir une carte d'état-major précise des forces en présence. C'est pourtant ce qui se passe dans la majorité des entreprises et dans la plupart des OPR lorsqu'il s'agit de développer ou de lancer une innovation.

De très nombreux auteurs ont proposé des dizaines de méthodes pour analyser les bases de données de brevets ([DEA 19], [JAF 17], [SQU 13]). Nous ne les citerons pas tous ici, car la quasi-majorité utilise des bases de données temporellement incomplètes, régionales, et insuffisamment nettoyées (voir les exemples sur la base Patstat de [KAN 16]). Les traductions automatiques utilisées sont souvent partielles et de qualité insuffisante notamment pour les brevets asiatiques. La base de brevets idéale devrait inclure tous les brevets issus de tous les Offices, même les plus petits. Travailler avec une base de brevets contenant des milliards de données nécessite des moyens informatiques adaptés et une parfaite maîtrise des règles de dépôts, de délivrance et de classification des brevets à l'échelle mondiale.

De rares professionnels sont cependant capables d'exploiter en profondeur ces informations. Les grands industriels recourent à des outils d'analyse massive et de visualisation de la distance sémantique entre les brevets afin de créer des cartes des technologies mondiales, de visualiser leur évolution secteur par secteur et de positionner les portefeuilles d'inventions par rapport à ceux des concurrents. Cette vision de type Google Earth (vue de haut avec possibilité de zoomer) permet d'analyser très finement la stratégie R\&D des entreprises et des OPR. Une telle vision apporte un avantage compétitif déterminant. La carte d'état-major des inventions est indispensable pour toute entreprise souhaitant développer une démarche d'Open innovation efficace et/ou anticiper le développement de la R\&D de ses concurrents.

L'outil le plus performant aujourd'hui est celui proposé en licence par Clarivate Analytics. Il combine une base de donnée de brevets unique au monde (DWPI) et un algorithme permettant de classer les brevets en fonction de leurs distances sémantiques et de les visualiser sous forme de cartes topographiques. Il est cher et complexe à maîtriser ce qui en limite la dissémination aux très grandes entreprises et à quelques OPR. Pour le rendre plus accessible, l'INPI est le premier Office de brevets au monde à avoir proposé le positionnement des inventions des start-up, PME ou des OPR dans leur 
environnement mondial, à prix coûtant, afin de leur permettre de lever des fonds plus facilement en mettant en évidence le caractère innovant de leurs inventions brevetées.

Cet article a pour objet d'expliquer comment sont créées ces cartes d'état-major technologiques, comment les interpréter et en exploiter les résultats pour en tirer des informations stratégiques qui se transformeront en avantages compétitifs majeurs.

\section{Choix de la base de données de brevets et des algorithmes d'analyse et de visualisation}

L'analyse sémantique de ces bases de données peut porter sur différentes parties du brevet. Travailler seulement sur le résumé du brevet n'est généralement pas recommandé, car leur contenu est souvent intentionnellement abscons de manière à peu divulguer l'invention pour ne pas alerter la concurrence. De plus, le résumé est parfois mal traduit. C'est pourquoi nous avons eu recours à la base de donnée DWPI (Derwent World Patent Index). Ses caractéristiques sont uniques au monde. Elle est très utilisée par les Offices de brevets et les grandes entreprises, car le résumé de chaque nouveau brevet est réécrit et traduit en anglais par un des cinq cents experts de Clarivate Analytics de façon à lui donner du sens, de le classer sur la base d'une ontologie et d'un vocabulaire prédéfini. Travailler sur les résumés DWPI, en y ajoutant titre et revendications des brevets apporte donc une pertinence bien meilleure qu'avec n'importe quelle autre base de données. Chaque nouveau brevet est analysé afin de le rattacher comme nouveau membre à une famille existante si l'invention est déjà protégée ou, dans le cas contraire, devient le brevet de priorité d'une nouvelle famille. Une famille DWPI correspond à une invention.

Le logiciel de Clarivate Analytics que nous avons ensuite utilisé combine deux fonctionnalités :

- un algorithme basé sur la classique méthode de pondération TF-IDF (Term Frequency-Inverse Document Frequency) analyse les textes. Cette mesure statistique augmente le poids des mots rares (sauf s'ils n'apparaissent qu'une fois) et sous pondère les mots trop fréquents (sans sens particulier). Cette méthode développée par [JON 72] a depuis fait ses preuves, notamment pour la recherche sur les brevets [KEL 18]. Chaque famille de brevet(s) est caractérisée par une à quatre idées clé (vecteurs), selon la complexité de la famille de brevets analysée. Une classification bayésienne naïve est alors appliquée. Les vecteurs vont permettre de positionner les brevets les uns par rapport aux autres dans une représentation en $n$ dimensions, la proximité entre deux brevets étant proportionnelle à la similarité de leur contenu lexical ;

- l'algorithme de visualisation transforme ensuite cet espace à $n$ dimensions en carte topographique en deux dimensions, le relief représentant la densité des brevets basés sur le même concept. Ainsi, les différentes technologies d'un secteur donné sont précisément géolocalisées ainsi que les propriétaires des diverses inventions.

Un million de familles de brevets peuvent rapidement être analysés en même temps et chaque carte technologique permet d'en visualiser jusqu'à soixante mille.

\section{Carte 1 : inventions cosmétiques mondiales}

Cette carte topographique (figure 1) représente la répartition des trois cent mille inventions brevetées en cosmétique, sur une période de vingt ans, dans le monde. Sa lecture est simple : toutes les inventions basées sur le même concept s'empilent au même endroit. Plus leur nombre est grand, plus la montagne est haute (en blanc). Ce sont les applications où la compétition est la plus forte. Les courbes de niveaux permettent d'apprécier la densité en innovation. Dans les plaines (zones de couleur marron ou verte), le nombre d'inventions est moins élevé. Si le concept de l'invention est très nouveau, le brevet correspondant se trouvera dans la mer, car il n'y a pas assez de brevets similaires pour créer une île. La forme des îles n'a pas d'importance. Les axes n'ont pas de signification. Ce qui compte, c'est la proximité. Les mots-clés permettent d'identifier les applications. Quand ces mots-clés sont (C) 2021 ISTE OpenScience - Published by ISTE Ltd. London, UK - openscience.fr 
insuffisamment explicites, il est possible de sonder les montagnes et de lire les brevets qu'elles contiennent pour en préciser l'application.

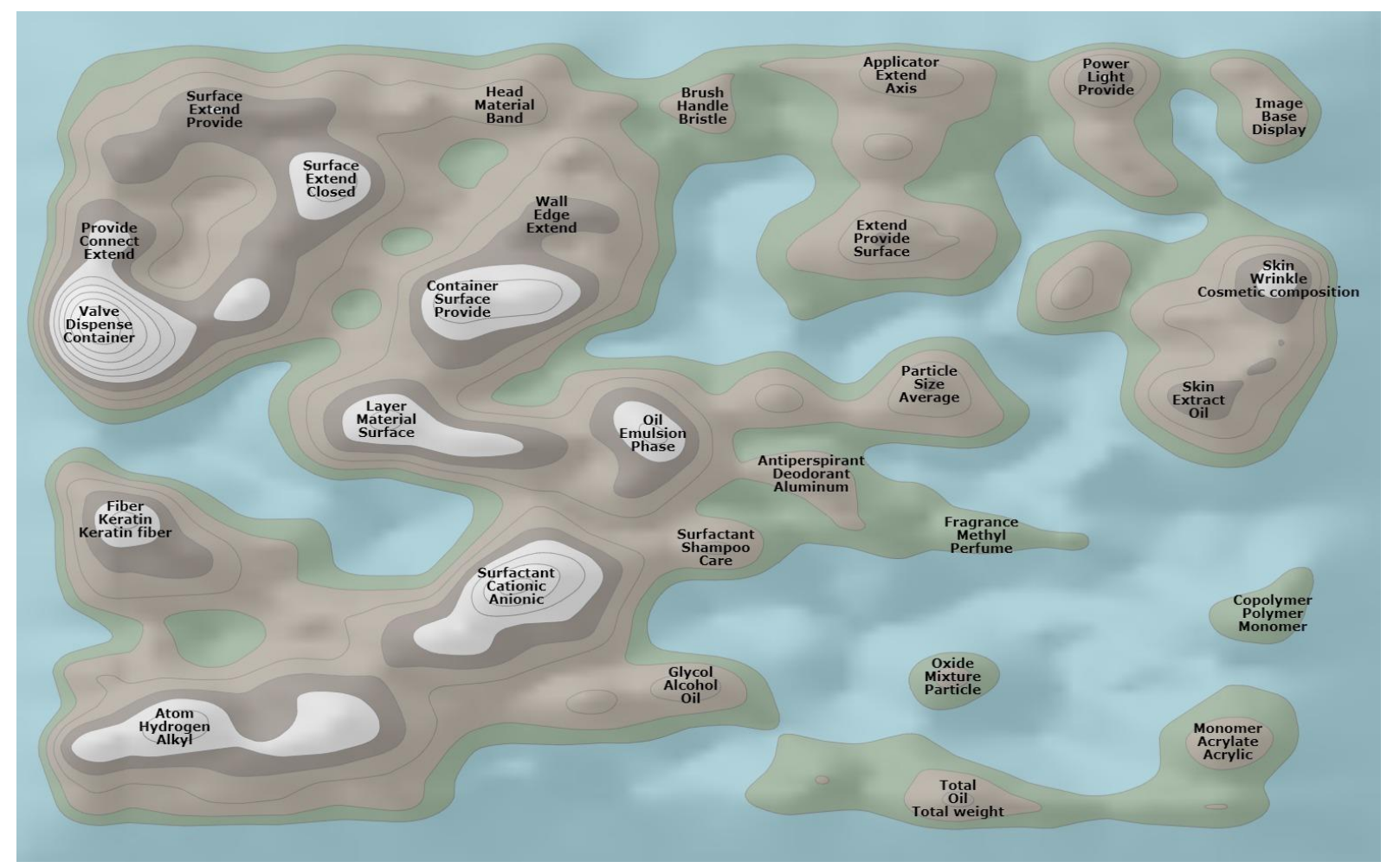

Figure 1. Inventions cosmétiques mondiales

Cette carte globale des inventions cosmétiques est très utile pour la direction R\&D de l'entreprise. Si l'une de ses inventions se positionne dans une zone à forte densité en innovation (montagne), il y a peu de chance qu'elle soit originale et encore moins qu'elle soit libre d'exploitation. Mieux vaudra abandonner le projet et collaborer avec l'entité propriétaire de l'invention recherchée.

Il est également possible de montrer les inventions brevetées sous-jacentes sous la forme de points colorés et d'accéder au texte de leur brevet par un simple clic.

\section{Carte 2 : détecter l'émergence d'une innovation}

La figure 2 montre les différentes applications de la technologie piézo-électrique (quarante mille inventions). Le donneur d'ordre voulait savoir s'il était possible d'utiliser cette technologie pour faire des sprays à gouttelettes ultra fines pour la cosmétique. Les ronds rouges dans la mer correspondent aux inventions de ce type. Les brevets ne sont pas encore assez nombreux pour constituer une île, mais ce sera le cas quelques mois plus tard. Réaliser des cartographies régulières du secteur où l'on opère permet donc d'identifier très précocement l'émergence d'innovations inédites. L'analyse des entreprises à l'origine de ces inventions permet ensuite de sélectionner la mieux adaptée pour une collaboration, voire de s'en inspirer pour déposer de nouveaux brevets.

\section{Cartes 3 à 5 : positionner ses brevets dans son environnement concurrentiel mondial}

Les start-ups ont généralement une vision très parcellaire, voire inexistante, de leur environnement en brevets, car les investisseurs prennent souvent le risque de les financer sans le vérifier. La figure 3 montre toutes les inventions du secteur audio et ses principales applications. Les inventions de la startup sont en vert et celles de son concurrent majeur, Dolby, en rouge. La plupart des inventions de la start-up sont dans la mer ou dans des zones peu denses en innovation. Elle est donc innovante. C'est ce type d'information qui rassure les investisseurs et les actionnaires des entreprises qu'elles que soient 
leurs tailles. Dans la case signal processing, on constate que deux brevets de la start-up (en vert) sont entourés de nombreux brevets de la société Dolby (en rouge).

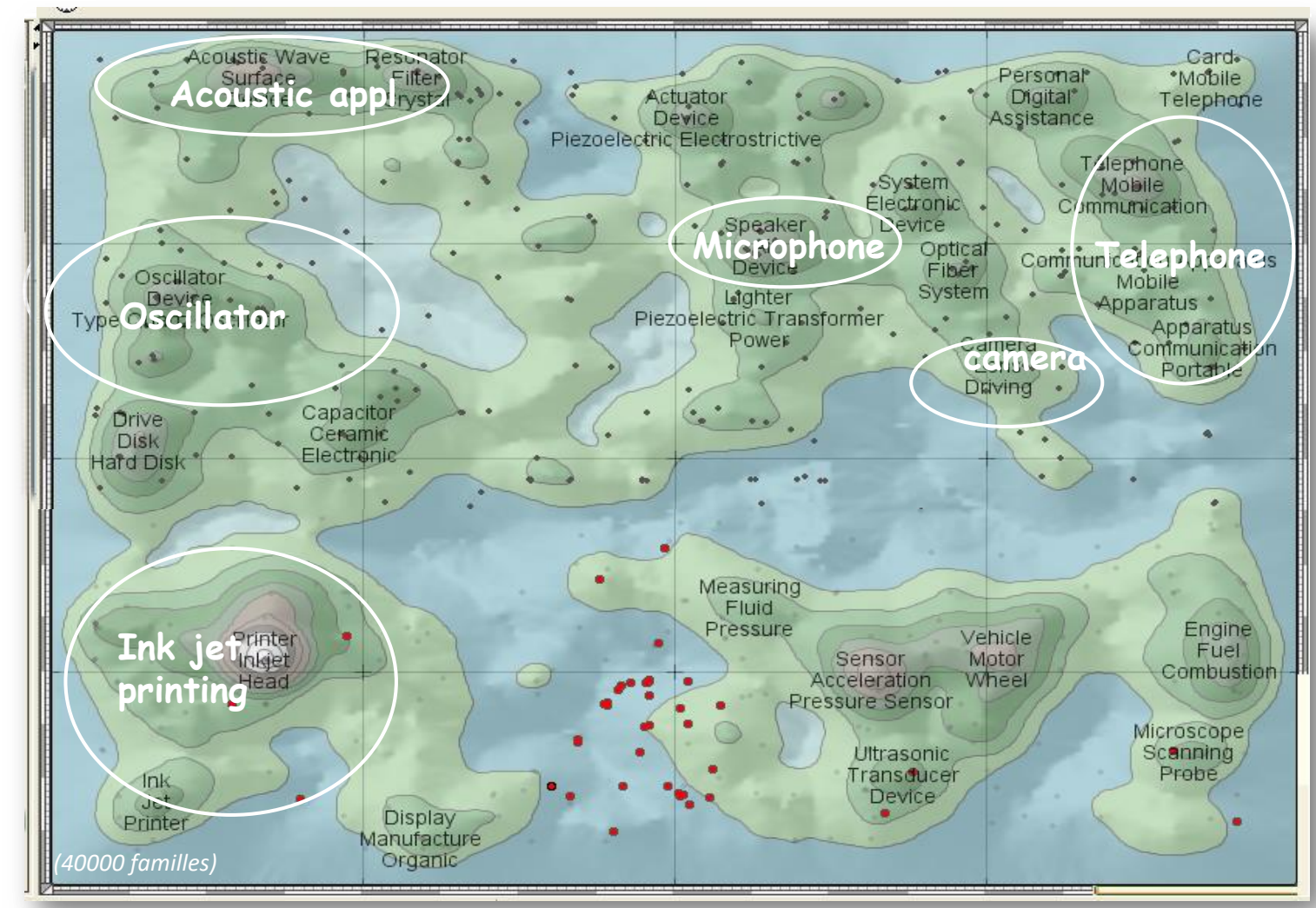

Figure 2. Détecter l'émergence d'une innovation

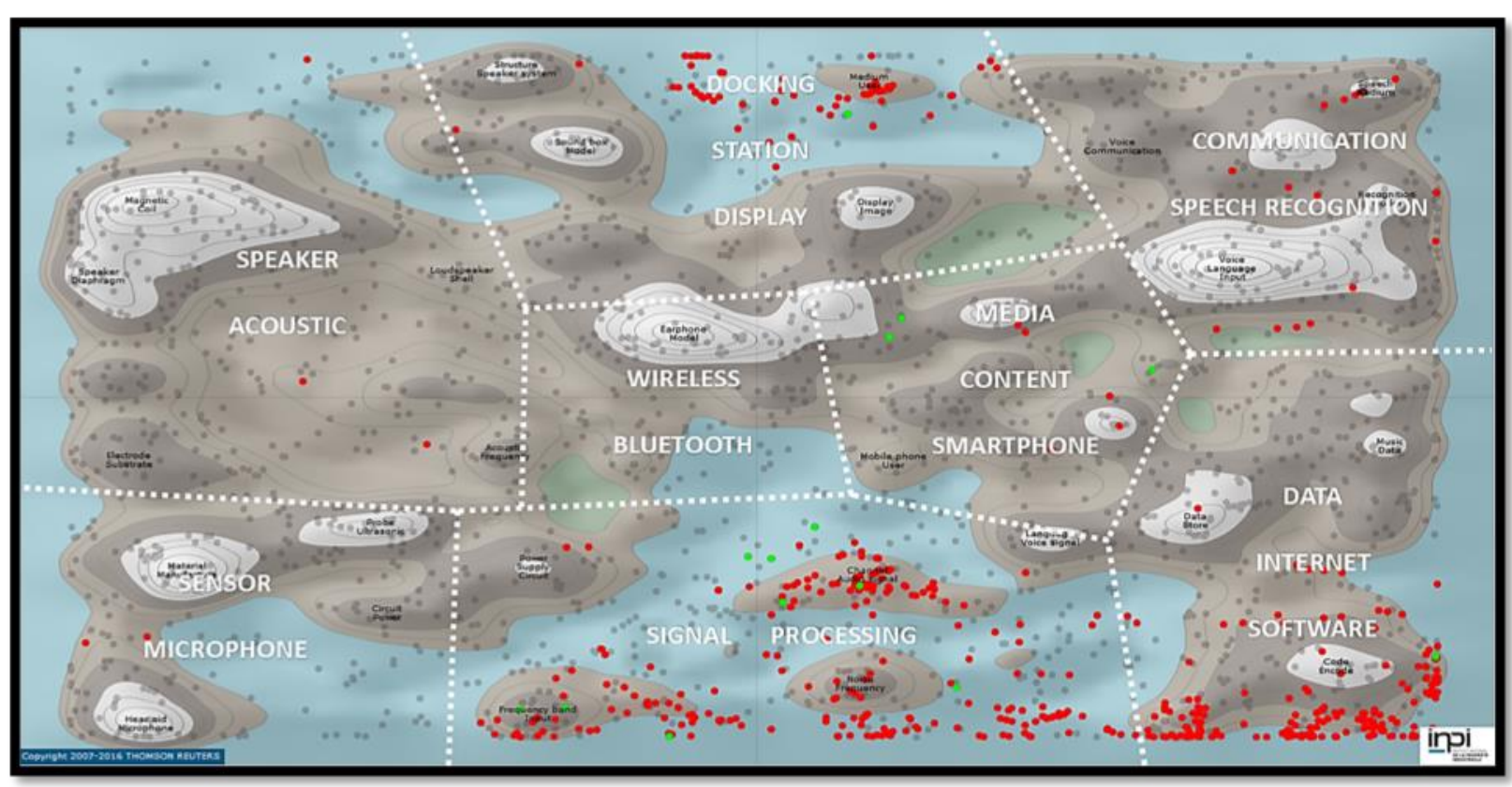

Figure 3. Positionner ses brevets dans son environnement concurrentiel mondial

Une telle situation risque de poser des problèmes majeurs de liberté d'exploitation à cette start-up et l'obliger à ne trouver un accord de licence qu'avec Dolby. En revanche, si les brevets de la start-up ont été déposés avant ceux de Dolby, c'est Dolby qui pourrait être obligé de négocier une licence avec la start-up. Une analyse juridique des brevets permettra de trancher et de prendre la bonne décision. 
La situation est cependant plus complexe que cela. En effet, seuls les brevets de Dolby ont été allumés alors que cette île est aussi constituée de brevets issus d'entités très différentes. Un zoom sur cette île (figure 4), centré sur le brevet vert de la start-up, montre qu'il existe plusieurs dizaines d'inventions très proches tout autour. L'extraction et l'analyse du texte de tous les brevets proches (points gris) permettra de positionner l'invention de cette start-up dans son environnement mondial, et pas seulement vis-à-vis de Dolby. L'analyse de cette liste permettra donc d'identifier de futurs licenciés, d'éventuels contrefacteurs, des partenaires potentiels ou de nouveaux risques de litiges. Il est très fréquent de rencontrer jusqu'à vingt pour-cent d'entités qui n'opèrent pas dans le secteur étudié, mais qui disposent de brevets dont les revendications peuvent s'avérer particulièrement gênantes pour les opérateurs du secteur.

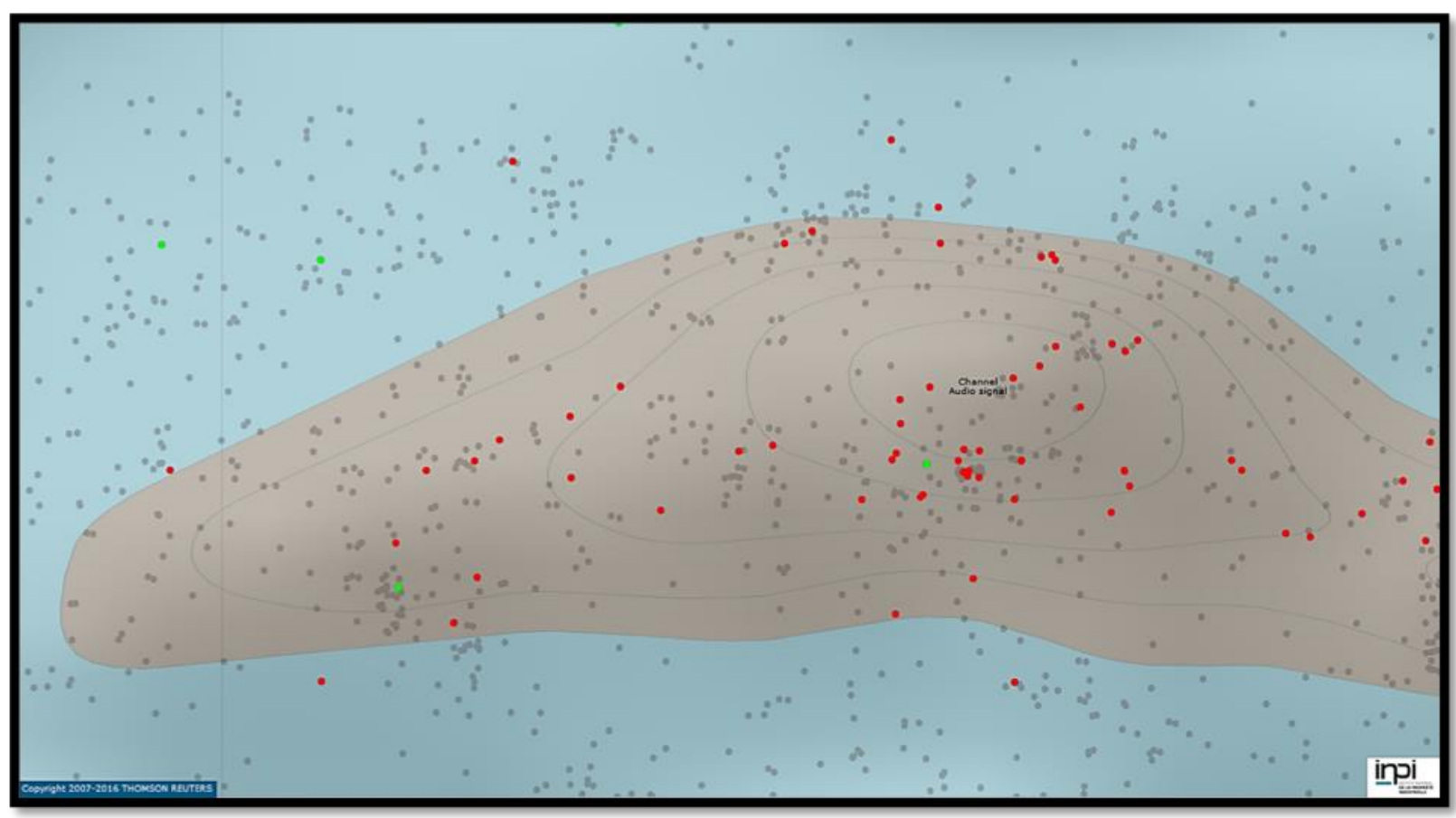

Figure 4. Positionner ses brevets dans son environnement concurrentiel mondial (bis)

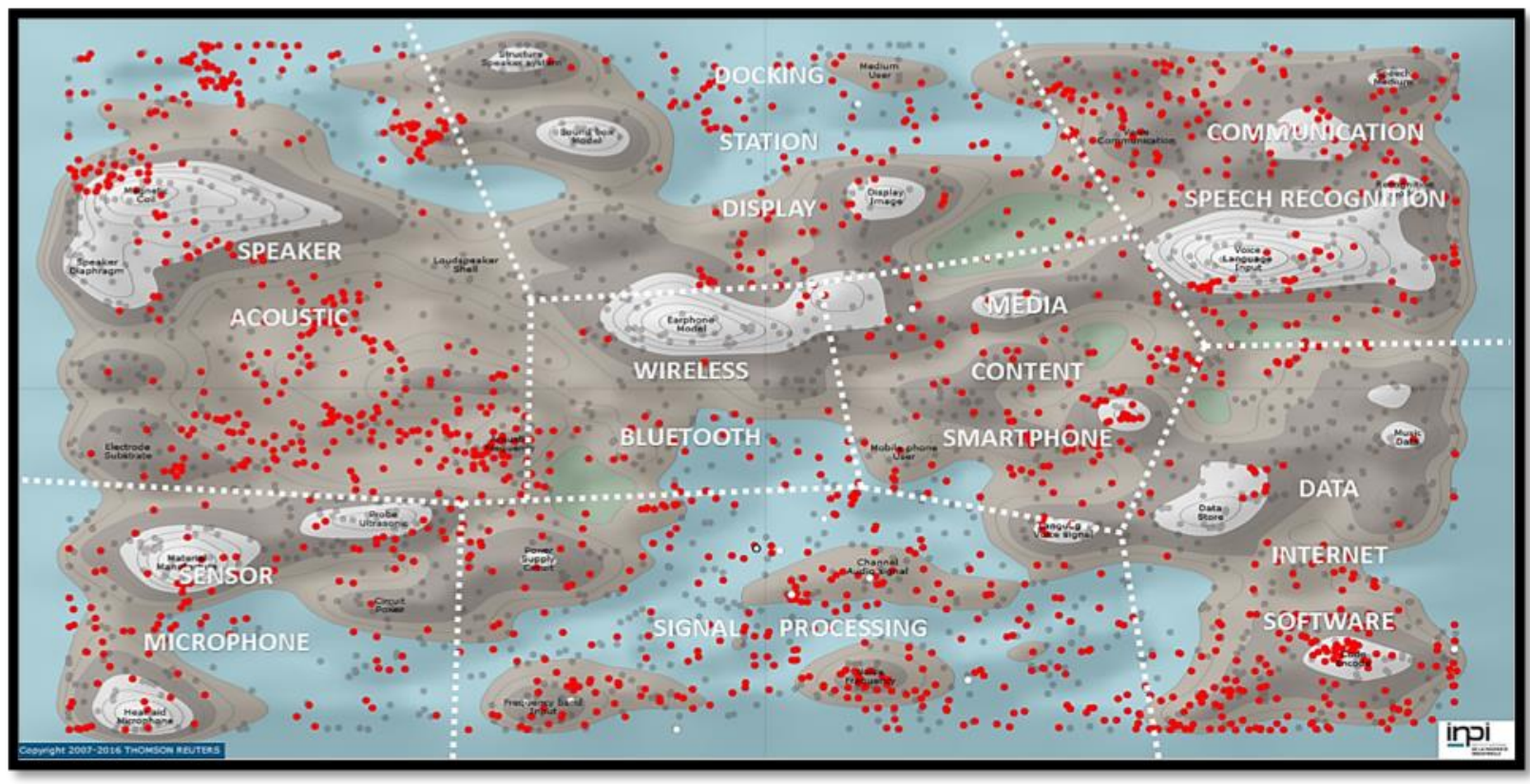

Figure 5. Rechercher un organisme public de recherche (OPR) pour accélérer son développement ou développer des inventions complémentaires 
Dans la figure 5, les inventions de la start-up sont représentées en blanc et les inventions des OPR mondiaux en rouge. Si on se focalise à nouveau sur l'île signal processing, il est facile de constater que de nombreuses opportunités de collaborations s'offrent à la start-up et qu'il est essentiel que ses chercheurs suivent de très près ces OPR.

\section{Cartes 6 et 7 : cartes d'état-major sectorielles}

Sur la figure 6, les inventions en vert sont celles de SEB et celles en rouge appartiennent à Dyson. L'électroménager est un secteur qui n'innove que lentement. Il est impressionnant de constater que Dyson a réussi en quelques années à créer de nouvelles applications et à les protéger par plusieurs grands et denses champs de mines de brevets afin de tenir ses concurrents à distance. On note la présence de brevets Dyson (gros ronds rouges) dans le territoire de SEB et réciproquement (gros ronds verts). C'est le principe du jeu de Go mais ces brevets sont insuffisamment nombreux pour perturber l'activité des deux entreprises. C'est un des rares exemples au monde où un challenger (Dyson) a attaqué un marché mature en créant de nouvelles inventions très différenciées et lourdement protégées, se constituant ainsi un avantage compétitif majeur. Une telle stratégie prend des années et il est étonnant que SEB n'ai pas davantage essayé de limiter la domination de Dyson dans les nouvelles applications.

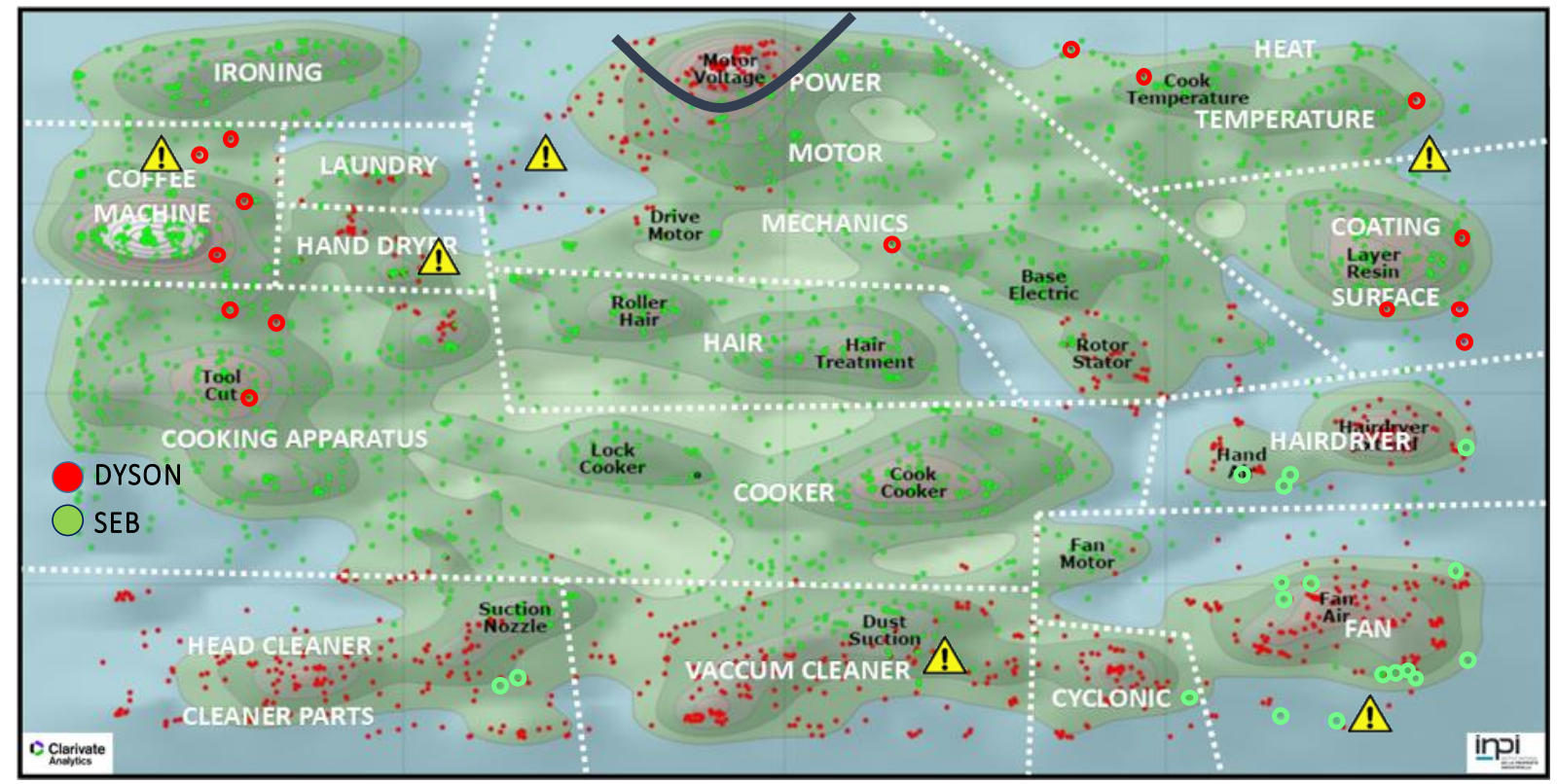

Figure 6. Carte d'état-major sectorielle - Electroménager

Lorsque deux entreprises veulent collaborer ensemble, elles demandent à leurs équipes $R \& D$ de définir les sujets d'intérêt potentiel. C'est un exercice extrêmement difficile et long notamment quand le portefeuille d'invention de chaque entreprise est constitué de plusieurs centaines voire de plusieurs milliers d'inventions. Rassembler les inventions des deux sociétés et créer une carte comme celle de SEB / Dyson prend moins d'une heure et permet de visualiser aisément toutes les zones où des collaborations potentielles pourraient s'établir (brevets portant sur des concepts proches).

Ces cartes sont également utiles en cas d'opérations de fusion / acquisition car la superposition des portefeuilles d'inventions montre immédiatement les zones de complémentarité des deux entreprises ou la volonté de diversification de l'entreprise qui prend l'initiative quand aucune complémentarité n'existe. Les zones de monopole résultant de la fusion sont également identifiables, ce qui a récemment conduit la Commission européenne à utiliser ces cartes dans l'opération de fusion entre Dow et Dupont. 
C'est typiquement la carte que chaque direction $R \& D$ devrait montrer à sa direction générale avant chaque grand projet d'investissement dans des projets d'innovations. Un général d'armée ne peut se battre sans savoir quelles forces sont en présence et où elles se tiennent. La carte de la figure 7 représente toutes les formulations cosmétiques brevetées dans le monde. Les codes couleur représentent des concurrents différents. Il est ainsi aisé d'identifier L'Oréal en rouge car c'est la seule société du secteur à pouvoir financer la création de champs de mines de brevets aussi importants et de se battre dans la plupart des applications restantes. Les autres concurrents créent des champs de mines plus modestes et plus disséminés. Certaines applications font l'objet d'un jeu de Go très agressif avec de multiples adversaires, comme par exemple, dans l'île Skin Cosmetic, en bas de l'image à gauche. La situation est très changeante au gré des acquisitions et des orientations stratégiques auxquelles elles répondent. Une telle carte est donc particulièrement utile aux directions générales.

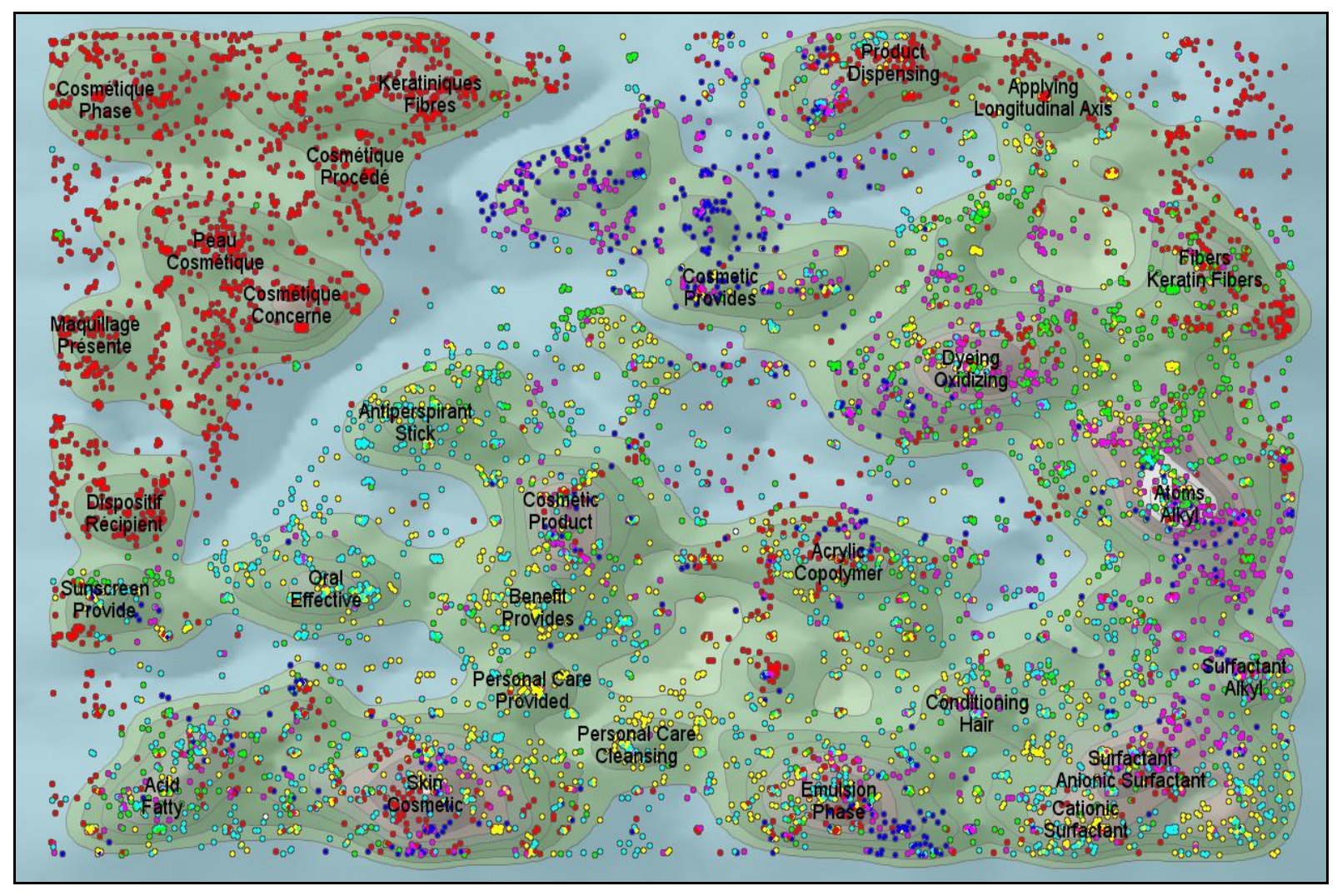

Figure 7. Carte d'état-major sectorielle - Cosmétiques

\section{Carte 8 : la cartographie des inventions au service de la stratégie des États}

$\mathrm{Au}$ lieu d'allumer les brevets d'entreprises spécifiques, il peut s'avérer particulièrement intéressant d'allumer tous les brevets déposés par les entreprises de chaque pays. Une carte combinant toutes les inventions des OPR et des entreprises d'un pays donné permet d'identifier facilement les applications stratégiques pour ce pays. La figure 8 montre les inventions pour chacune des applications du secteur de l'appareillage médical. Les inventions vertes sont chinoises, les rouges sont américains et les jaunes sont européennes. De manière inattendue, on constate que certains pays ont créé d'immenses champ de mines afin de s'accaparer la commercialisation de certaines applications. C'est ainsi que les américains dominent le secteur des dispositifs implantables et des cathéters. Plus étonnant est la focalisation des inventions chinoises sur la transmission des données. Une telle démarche résulte d'une stratégie volontariste de ce pays, car tous les appareils médicaux doivent communiquer, ce qui pourrait obliger la plupart des fabricants d'appareils à obtenir des licences de sociétés chinoises. Ce n'est cependant pas encore le cas, car les entreprises chinoises ne brevettent quasiment qu'en Chine ... mais la situation évolue très vite vers l'internationalisation des meilleurs brevets. 
8. Carte 9 : la cartographie des inventions au service des collaborations entre États ou de l'implantation des entreprises dans de nouveaux pays - exemple de la Corée du sud

S'implanter dans un nouveau pays est toujours un risque si une analyse approfondie des brevets locaux n'a pas été réalisée avant d'y investir. C'est une étude souvent complexe et chère à réaliser, car les brevets d'un pays sont généralement très nombreux et dans une langue souvent difficile à traduire. La solution réside dans la création d'une carte rassemblant tous les brevets déposés par les entreprises et OPR du pays étudié. À titre d'exemple, vous trouverez la carte des inventions coréennes en figure 9. Si l'on superposait les brevets de l'entreprise qui souhaite s'implanter ou commercer avec la Corée du Sud, il serait facile d'identifier les brevets coréens dont le concept est similaire à de ceux de l'entreprise, de les extraire et d'identifier leurs propriétaires, leur couverture géographique et la portée de chacune des inventions revendiquées ... avant de les rencontrer. Nanti de telles informations, le risque de se déplacer pour rien, et de dévoiler sa stratégie confidentielle à l'une des entreprises est beaucoup plus limité, car l'étude préalable des partenaires potentiels permet de faire moins d'erreurs et ainsi, de maximiser les chances d'un partenariat réussi, basé sur des informations plus précises.

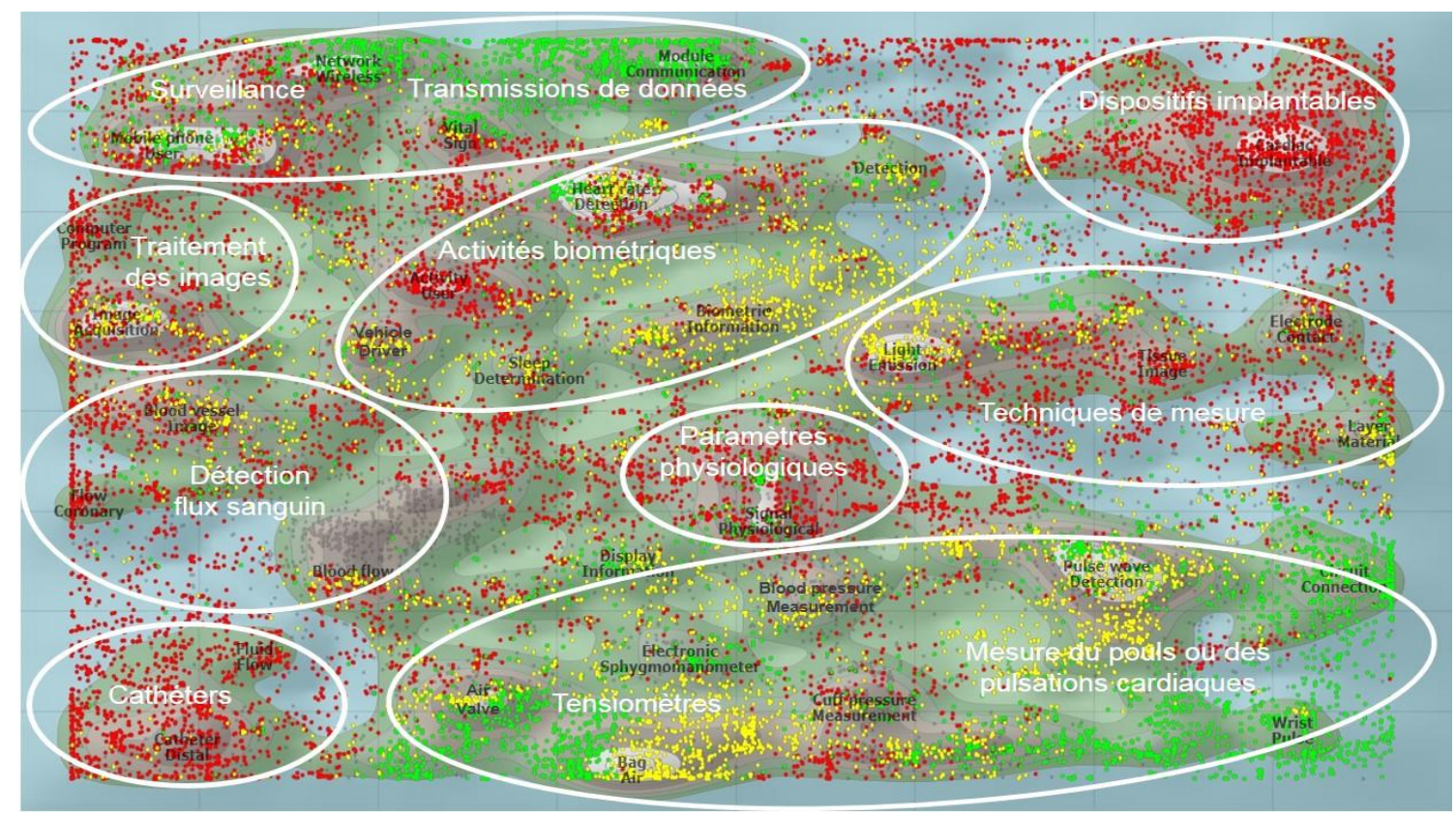

Figure 8. La cartographie des inventions au service de la stratégie des Etats

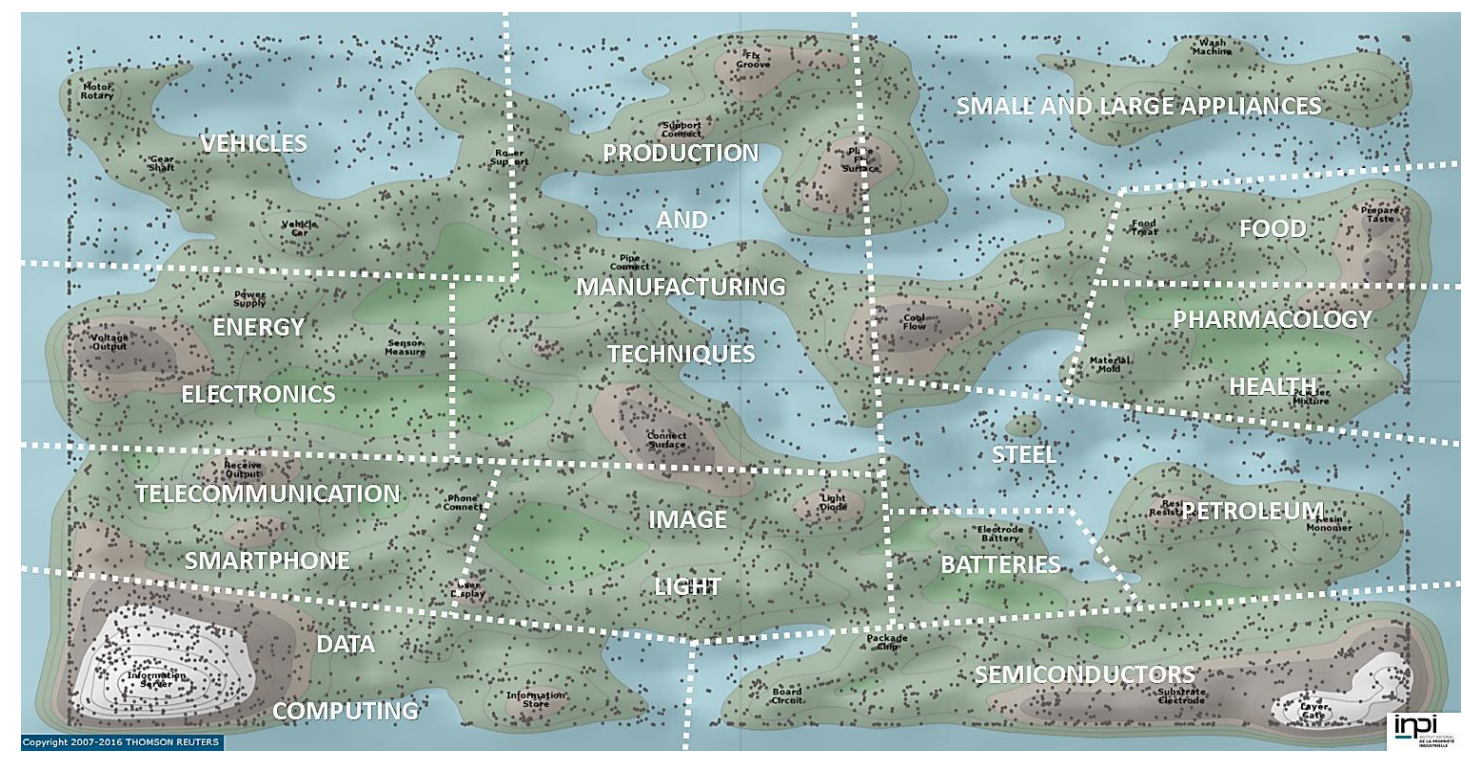

Figure 9. La cartographie des inventions au service des collaborations entre États ou de l'implantation des entreprises dans de nouveaux pays 
La démarche est la même pour déterminer les domaines potentiels de collaborations entre deux pays. La cartographie des inventions est la seule façon de conduire rapidement une analyse exhaustive des options disponibles, mais elle n'a, à notre connaissance, jamais été utilisée de cette manière, très probablement, car les dirigeants des offices gouvernementaux ne savent pas que de tels outils existent.

\section{Carte 10 : La cartographie permet la comparaison des stratégies de recherche entre pays}

La figure 10 montre les inventions brevetées basées sur l'ADN recombinant dans la recherche publique. Toutes les inventions européennes sont prises en compte mais seules celles de la France (bleu) de la Grande-Bretagne (rouge) et de l'Allemagne (jaune) sont représentées. Si l'on regarde les applications agricoles, il est facile de constater que l'Allemagne domine en créant plusieurs champs de mines de brevets, ce qui montre leur domination dans plusieurs applications. Les Français et les Anglais ont tendance à jouer au jeu de Go sans se préoccuper de créer leurs propres champs de mines de brevets. Les chercheurs français semblent créatifs si l'on en juge par le nombre de brevets déposés, mais ils ne cherchent pas à renforcer leurs positions en déposant des brevets complémentaires. Cela signe soit un manque de moyens financiers soit l'absence de politique industrielle pour que la France dispose de technologies incontournables. Une étude sur les inventions des SATT en France (non publiée) montrait une stratégie similaire.

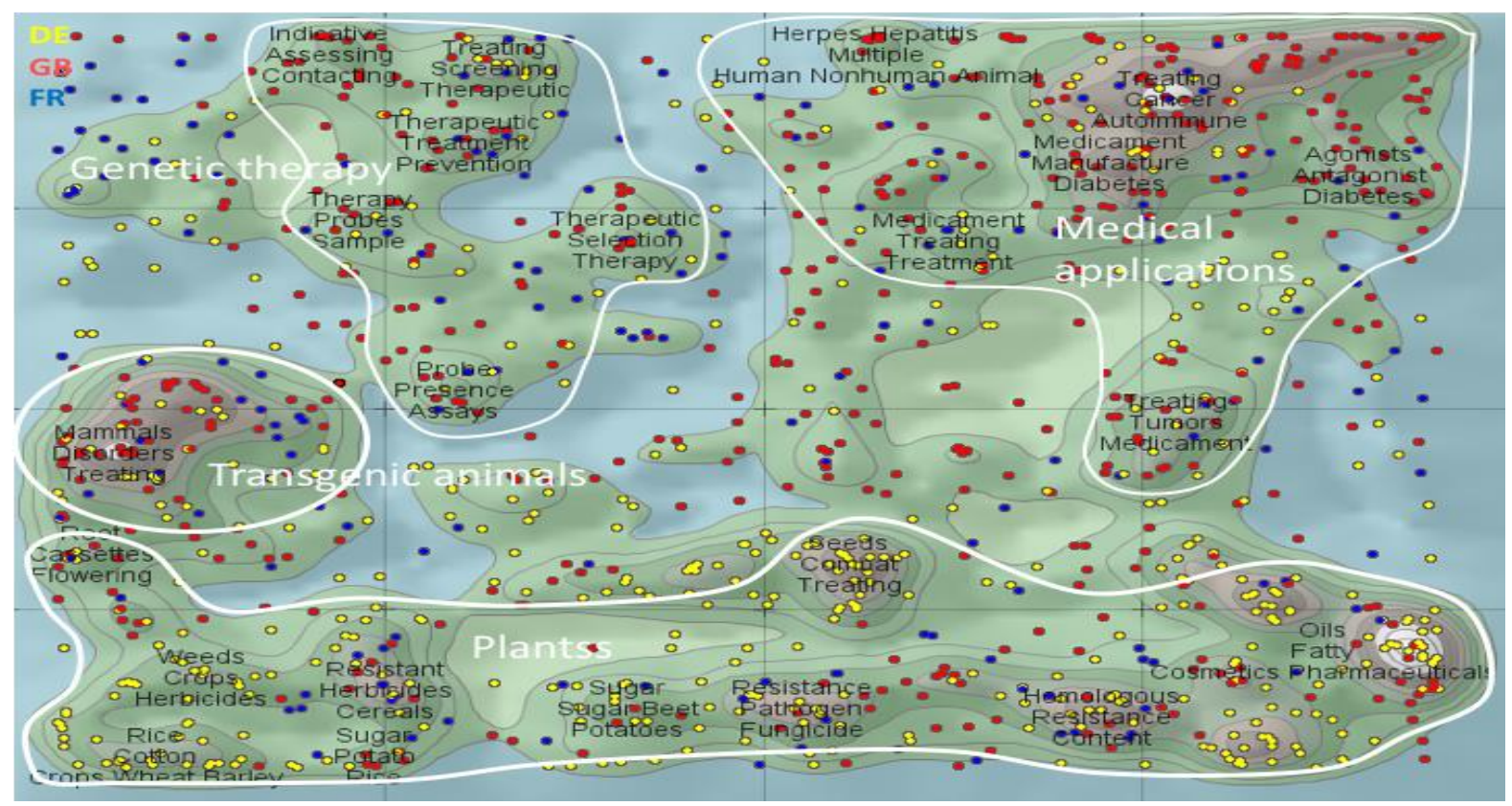

Figure 10. La cartographie permet la comparaison des stratégies de recherche entre pays

\section{Discussion et conclusion}

La cartographie permet de positionner les inventions dans leur environnement mondial sur une carte topographique. Cette visualisation permet de mieux anticiper, de mieux investir, d'accroître la productivité de la $R \& D$ et d'optimiser les conditions de sélection des partenariats avec des entreprises ou des OPR. C'est un outil indispensable pour l'Open innovation, car il permet d'identifier de multiples opportunités et de variantes de la technologie recherchée. Cette carte d'état-major de la technologie mondiale est particulièrement appréciée par les directions générales, car elle permet de visualiser les progrès de la recherche dans tous les secteurs, l'activité des concurrents et les tendances de la recherche mondiale. D'autres outils permettent de créer des cartographies, mais aucun n'égale celui que nous avons étudié en raison de la qualité de la réécriture des résumés de chacun des brevets nouvellement déposés. Plusieurs tentatives sont en cours afin d'utiliser l'intelligence artificielle pour mieux mesurer la distance sémantique entre deux brevets. À notre connaissance, aucune n'a encore abouti, hormis sur de petits échantillons ou sur des questions de classifications assez banales. La 
principale raison de cet échec tient à la façon dont sont écrits les brevets, notamment résumés et revendications. Il s'agit d'un jargon volontairement travaillé pour divulguer le moins possible et induire en erreur les compétiteurs sur les intentions réelles du déposant. Surmonter ces obstacles serait sûrement possible, mais cela n'est ni l'intérêt de nombreux et puissants déposants, ni l'intérêt des Offices de brevets qui emploient des milliers d'experts pour délivrer les brevets. Cet outil devrait donc rester unique pendant encore quelques années.

La création d'une carte répond à une question précise. Plusieurs jours peuvent être nécessaires pour choisir la carte la plus parlante et en valider le paysage auprès d'experts du secteur. Si les scientifiques analysent la portée et l'originalité des inventions, ce sont les stratèges de la R\&D, des unités opérationnelles ou de la direction générale qui seront à même d'interpréter finement les données issues des cartes pour en tirer des décisions stratégiques. La confrontation de ces données avec toutes celles recueillies par l'entreprise permet de créer une vision plus proche de la réalité du terrain. L'environnement technologique devient beaucoup plus transparent qu'auparavant. Cela permet de développer l'Intelligence Technologique d'une entreprise.

La plupart des articles scientifiques ne parlent que rarement des outils développés par l'industrie alors que certains scientifiques tentent de développer des outils similaires. Ce n'est malheureusement pas la beauté de l'algorithme ou de la démonstration qui compte, mais la robustesse et la validation de l'outil. Travailler avec un million de brevets " propres » et régionaux n'est pas la même chose que travailler avec une base de cent trente millions de brevets dans toutes les langues. La robustesse des méthodes proposées n'est quasiment jamais abordée sérieusement. Un outil n'est rien sans validation des résultats obtenus par des opérationnels du secteur. Ce qui est montré par l'outil est-il vrai ? La distance sémantique entre les brevets est-elle pertinente notamment lorsqu'elle est visualisée ? Cette validation est essentielle, mais elle prend beaucoup de temps et nécessite beaucoup de moyens. Une collaboration avec un Office de brevets comme l'INPI ou une grande entreprise du secteur est idéale.

Notre base de trois cent mille brevets « Cosmétiques » est bien caractérisée en raison de nos vingt ans d'expertise du secteur et de nos liens privilégiés avec le leader mondial du secteur. Nous avons par ailleurs mesuré la qualité technologique des inventions des dix plus gros déposants du secteur. Cette base est à la disposition de tous ceux qui souhaiteraient y avoir accès.

\section{Bibliographie}

[CAI 14] CAILlaud F., MenIERE Y., « Strategic intelligence on patents », in Cambridge university Press book on Patents markets in the global knowledge economy: theory, empirics and public policy implications, 2014.

[CH0 18] CHO Y., KIRKEWOOG S., DAIM T.U., « Managing strategic intellectual property assets in the fuzzy front end of new product development process », R\&D Management, vol. 48, p. 354-374, 2018.

[DEA 19] DE AlCANTARA D.P., MARTENS M.L., «Technology Roadmapping (TRM): a systematic review of the literature focusing on models », Technological Forecasting and Social Change, vol. 138, p. 127-138, 2019.

[JAF 17] JAFFE A.B., DE RASSENFOSSE G., « Patent citation data in social science research: overview and best practices », Journal of the Association for Information Science and Technology, vol. 68, p. 1360-1374, 2017.

[JON 72] JONES K.S., «A statistical interpretation of term specificity and its application in retrieval », Journal of Documentation, https://doi.org/10.1108/eb026526, 1972.

[KAN 16] KANG B., TARASCONI G., «PATSTAT revisited: suggestions for better usage », World Patent Information, https://doi.org/10.1016/j.wpi.2016.06.001, 2016.

[Kel 18] Kelly B.T., Papanikolaou D., Seru A., TADDy M., «Measuring technological innovation over the long run », SSRN Electronic Journal, https://doi.org/10.2139/ssrn.3279254, 2018.

[MCM 03] MCMillan G.S., MAURI A., HaLMiLton R.D., «The impact of publishing and patenting activities on new product development and firm performance: the case of the US pharmaceutical industry », International Journal of Innovation Management, vol. 7, p. 213-221, 2003. 
[SQU 13] SQUICCIARINI M., DERnIS H., CRISCUOlo C., « Measuring patent quality », OECD Science, Technology and Industry Working Papers, https://doi.org/10.1016/j.ajodo.2004.10.009, 2013.

[VAN 08] VAn Pottelsberghe De La Potterie B., VAn Zeebroeck N., «A brief history of space and time: the scope-year index as a patent value indicator based on families and renewals », Scientometrics, vol. 75, p. 319-338, 2008.

[WIP 18] WIPO, Guide to the International Patent Classification, http://www.wipo.int/classifications/ipc/, 2018. 